\title{
Existence and uniqueness solution of a class of quasilinear parabolic boundary control problems
}

\author{
M. H. FARAG ${ }^{1}$, T. A. TAlaAT ${ }^{2}$ And E. M. Kamal ${ }^{3}$ \\ Minia University, \\ Department of Mathematics, Faculty of Science, Minia, Egypt. \\ farag5358@yahoo.com ${ }^{1}$ \\ talaat.20080yahoo. $\mathrm{com}^{2}$ \\ esamkamal550yahoo. $\mathrm{com}^{3}$
}

\begin{abstract}
This paper presents an optimal control of processes described by a quasilinear parabolic systems with controls in the coefficients of equation, in the boundary condition and in the right side of this equation. Theorems concarning the existence and uniqueness for the solution of the cosidering problem are invistigated.
\end{abstract}

\section{RESUMEN}

Este artículo presenta un control óptimo de procesos descritos por un sistema parabólico cuasilineal con control en los coeficientes de la ecuación, en la condición de frontera y en el lado derecho de esta ecuación. Se investigan los teoremas relacionados con la existencia y unicidad para la solución del problema considerado

Keywords and Phrases: Optimal control, Quasilinear Parabolic Equation, Existence and Uniquness Theorems.

2010 AMS Mathematics Subject Classification: 49J20, 49K20, 49M29, 49M30 


\section{Introduction}

Optimal control problems for partial differential equations are currently of much interest. A larage amount of the theoretical concept which governed by quasilinear parabolic equations [1-5] has been investigated in the field of optimal control problems. These problems have dealt with the processes of hydro- and gasdynamics, heatphysics, filtration, the physics of plasma and others [6-8]. The study and determination of the optimal regimes of heat conduction processes at a long interval of the change of temperture gives rise to optimal control problems with respect to a quasilinear equation of parabolic type. In this work, we consider a constrained optimal control problem with respect to a quasilinear parabolic equation with controls in the coefficients of the equation. The existence and uniqueness of the optimal control problem is proved.

\section{Statement of the problem}

Let $\mathrm{D}$ is a bounded domain of the $\mathrm{N}$-dimensional Euclidean space $\mathrm{E}_{\mathrm{N}} ; \Gamma$ be the boundary of $\mathrm{D}$, assumed to be sufficiently smooth; $v$ is the exterior unit normal of $\Gamma$; $\mathrm{T}>0$ be a fixed time ; $\Omega=\mathrm{D} \times(0, \mathrm{~T}] ; \mathrm{S}=\Gamma \times(0, \mathrm{~T}]$.

Now we consider a class of optimal control problems governed by the following quasilinear parabolic system.

$$
\begin{gathered}
L(v) y(x, t)=f\left(x, t, v_{2}\right),(x, t) \in \Omega, \\
y(x, 0)=\phi(x), x \in D, \\
\left.\sum_{i=1}^{n} \lambda_{i}\left(y, v_{0}\right) \frac{\partial y}{\partial x_{i}} \cos \left(v, x_{i}\right)\right|_{S}=g(\zeta, t),(x, t) \in S
\end{gathered}
$$

where $\phi \in \mathrm{L}_{2}(\mathrm{D}), \mathrm{g}(\zeta, \mathrm{t}) \in \mathrm{L}_{2}(\mathrm{~S})$ are given functions and the differential operator $\mathrm{L}$ takes the following form:

$$
L(v) z(x, t)=\frac{\partial z}{\partial t}-\sum_{i=1}^{n} \frac{\partial}{\partial x_{i}}\left[\lambda_{i}\left(z, v_{0}\right) \frac{\partial z}{\partial x_{i}}\right]+\sum_{i=1}^{n} B_{i}\left(z, v_{1}\right) \frac{\partial z}{\partial x_{i}}
$$

$y(x, t), v=\left(v_{0}, v_{1}, v_{2}\right)$ are the state and the controls rspectively for the system (1).

Furthermore, we consider the functional of the form

$$
J_{\beta}(v)=\int_{S}\left[y(\zeta, t)-f_{0}(\zeta, t)\right]^{2} d \zeta d t+\beta \sum_{m=0}^{2}\left\|v_{m}-\omega_{m}\right\|_{l_{2}}^{2},
$$

which is to minimized under condition (1) and additional restricitions

$$
v_{0} \leq \lambda_{i}\left(y, v_{0}\right) \leq \mu_{0}, v_{1} \leq B_{i}\left(y, v_{1}\right) \leq \mu_{1}, r_{1} \leq y(x, t) \leq r_{2}, i=\overline{1, n}
$$


over the class

$$
V=\left\{v=\left(v_{0}, v_{1}, v_{2}\right): v_{m}=\left(v_{0 m}, v_{1 m}, \cdots, v_{i m}, \cdots\right) \in l_{2},\left\|v_{m}\right\|_{l_{2}} \leq R_{m}, m=\overline{0,2}\right\}
$$

and $f_{0}(\zeta, t) \in L_{2}(S)$ is a given function and $\beta \geq 0, v_{j}, \mu_{j}, j=1,2, r_{1}, r_{2}, R_{m}>0$ are positive numbers, $\omega_{m}=\left(\omega_{0 m}, \omega_{1 m}, \cdots, \omega_{i m}, \cdots\right) \in l_{2}, m=\overline{0,2}$ are given numbers.

Throughout this paper, we adopt the following assumptions.

Assumption 2.1: $V$ is closed and bonded subset of $l_{2}$.

Assumption 2.2: The functions $B_{i}\left(y, v_{1}\right), \lambda_{i}\left(y, v_{0}\right), i=\overline{1, n}$ are continuous on $(y, v) \in$ $\left[r_{1}, r_{2}\right] \times l_{2}$ have continuous derivatives in $y$ at $\forall(y, v) \in\left[r_{1}, r_{2}\right] \times l_{2}$ and $\frac{\partial B_{i}}{\partial y}, \frac{\partial \lambda_{i}}{\partial y}, i=\overline{1, n \text { are }}$ bounded.

Assumption 2.3: The function $f\left(x, t, v_{2}\right)$ is given function continuous in $v_{2}$ on $l_{2}$ for almost all $(x, t) \in \Omega$, bounded and measurable in $x, t$ on $\Omega \forall v_{2} \in l_{2}$.

Assumption 2.4: The functions $B_{i}\left(y, v_{1}\right), \lambda_{i}\left(y, v_{0}\right), i=\overline{1, n}, f\left(x, t, v_{2}\right)$ satisfy a Lipschitz condition for $v_{1}, v_{0}, v_{2}$, then

$$
\begin{gathered}
\left|B_{i}\left(y(x, t), v_{1}+\delta v_{1}\right)-B_{i}\left(y(x, t), v_{1}\right)\right| \leq S_{0}(x, t)\left\|\delta v_{1}\right\|_{l_{2}}, i=\overline{1, n} \\
\left|\lambda_{i}\left(y(x, t), v_{0}+\delta v_{0}\right)-\lambda_{i}\left(y(x, t), v_{0}\right)\right| \leq S_{1}(x, t)\left\|\delta v_{0}\right\|_{l_{2}, i}=\overline{1, n} \\
\left|f\left(x, t, v_{2}+\delta v_{2}\right)-f\left(x, t, v_{2}\right)\right| \leq S_{2}(x, t)\left\|\delta v_{2}\right\|_{l_{2}}
\end{gathered}
$$

for almost all $(x, t) \in \Omega, \forall y \in\left[r_{1}, r_{2}\right], \forall v_{m}, v_{m}+\delta v_{m} \in l_{2}$ such that $\left\|v_{m}\right\|_{l_{2}},\left\|v_{m}+\delta v_{m}\right\|_{l_{2}} \leq R_{m}$ where $S_{m}(x, t) \in L_{\infty}, m=\overline{0,2}$.

Assumption 2.5: The first derivatives of the functions $B_{i}\left(y, v_{0}\right), \lambda_{i}\left(y, v_{0}\right), i=\overline{1, n}$ and $f\left(x, t, v_{2}\right)$ with respect to $v$ are continuous functions in $\left[r_{1}, r_{2}\right] \times l_{2}$ and for any $v_{m} \in l_{2}$ such that $\left\|v_{\mathrm{m}}\right\|_{l_{2}} \leq \mathrm{R}_{\mathrm{m}}, \mathrm{m}=\overline{0,2}$.

Definition 2.1: The problem of finding the function $y=y(x, t) \in V_{2}^{0,1}(\Omega)$ from condition (1)-(2) at given $v \in \mathrm{V}$ is called the reduced problem.

Definition 2.2: A function $y=y(x, t) \in V_{2}^{1,0}(\Omega)$ is said to be a solution of the problem 
(1)-(2), if for all $\eta=\eta(x, t) \in W_{2}^{1,1}(\Omega)$ the equation

$$
\begin{aligned}
& \int_{\Omega}\left[-y \frac{\partial \eta}{\partial t}+\sum_{i=1}^{n} \lambda_{i}\left(y, v_{0}\right) \frac{\partial y}{\partial x_{i}} \frac{\partial \eta}{\partial x_{i}}-\sum_{i=1}^{n} B_{i}\left(y, v_{1}\right)\left(\frac{\partial y}{\partial x_{i}}\right) \eta(x, t)\right. \\
& \left.-f\left(x, t, v_{2}\right) \eta(x, t)\right] d x d t=\int_{D} \phi(x) \eta(x, 0) d x+\int_{S} g(\zeta, t) \eta(\zeta, t) d \zeta d t,
\end{aligned}
$$

is valid and $\eta(x, T)=0$.

It is proved in [8] that, under the foregoing assumptions, a reduced problem (1)-(2) has a unique solution and $\left|\frac{\partial y}{\partial x_{i}}\right| \leq C_{1}, i=\overline{1, n}$ almost at all $(x, t) \in \Omega, \forall v \in V$, where $C_{1}$ is a certain constant.

\section{The Existence Theorem}

Optimal control problems of the coefficients of differential equations do not always have solution [9]. Examples in [10] and elswhere of problems of the type (1)-(4) having no solution for $\beta=0$. A problem of minimization of a functional is said to be unstable, when a minimizing sequare does not converge to an element minimizing the functional [6].

To begin with, we need

Theorem 3.1 Under the above assumptions for every solution of the reduced problem (1)-(2) the following estimate is valid:

$$
\|\delta y\|_{V_{2}^{1,0}(\Omega)} \leq C_{2}\left[\left\|\sqrt{\sum_{i=1}^{n}\left(\Delta \lambda_{i} \frac{\partial y}{\partial x_{i}}\right)^{2}}\right\|_{L_{2}(\Omega)}+\left\|\Delta f-\sum_{i=1}^{n} \Delta B_{i} \frac{\partial y}{\partial x_{i}}\right\|_{L_{2}(\Omega)}\right],
$$

where $\delta y(x, t)=y(x, t ; v+\delta v)-y(x, t ; v), \delta y(x, t) \in W_{2}^{1,1}(\Omega), \Delta \lambda_{i}=\lambda_{i}\left(u, v_{0}+\delta v_{0}\right)-\lambda_{i}\left(u, v_{0}\right)$ $, \Delta \mathrm{B}_{\mathfrak{i}}=\mathrm{B}_{\mathfrak{i}}\left(\mathrm{u}, v_{1}+\delta v_{1}\right)-\mathrm{B}_{\mathfrak{i}}\left(\mathrm{u}, v_{1}\right), \Delta f=f\left(x, t, v_{2}+\delta v_{2}\right)-f\left(x, t, v_{2}\right)$ and $\mathrm{C}_{2} \geq 0$ is a constant not dependent on $\delta v=\left(\delta v_{0}, \delta v_{1}, \delta v_{2}\right), \delta v_{m} \in l_{2}, m=\overline{0,2}$.

\section{ptoof}

Set $\delta y(x, t)=y(x, t, v+\delta v)-y(x, t ; v), y=y(x, t ; v), \bar{y}=y(x, t ; v+\delta v)$. From (5) it follows that

$$
\begin{gathered}
\int_{\Omega}\left[-\delta y \frac{\partial \eta}{\partial t}+\sum_{i=1}^{n} \overline{\lambda_{i}} \frac{\partial \delta y}{\partial x_{i}} \frac{\partial \eta}{\partial x_{i}}+\sum_{i=1}^{n} \frac{\partial \lambda_{i}\left(y+\theta_{1 i}, v_{0}+\delta v_{0}\right)}{\partial y} \frac{\partial y}{\partial x_{i}} \frac{\partial \eta}{\partial x_{i}} \delta y\right. \\
+\sum_{i=1}^{n} \Delta \lambda_{i} \frac{\partial y}{\partial x_{i}} \frac{\partial \eta}{\partial x_{i}}+\sum_{i=1}^{n} \overline{B_{i}} \frac{\partial \delta y}{\partial x_{i}} \eta+\sum_{i=1}^{n} \Delta B_{i}\left(\frac{\partial y}{\partial x_{i}}\right) \eta \\
\left.-\sum_{i=1}^{n} \frac{\partial B_{i}\left(y+\theta_{2 i}, v_{1}+\delta v_{1}\right)}{\partial y} \frac{\partial y}{\partial x_{i}} \delta y \eta-\Delta f \eta\right] d x d t=0
\end{gathered}
$$


for all $\eta=\eta(x, t) \in W_{2}^{1,1}(\Omega)$ and $\eta(x, T)=0$.

Here $\theta_{1 i}, \theta_{2 i} \in(0,1), i=\overline{1, n}$ is some number, $\overline{\lambda_{i}} \equiv \lambda_{i}\left(y+\delta y, v_{0}+\delta v_{0}\right), \Delta \lambda_{i} \equiv \lambda_{i}\left(y, v_{0}+\right.$ $\left.\delta v_{0}\right)-\lambda_{i}\left(y, v_{0}\right), \overline{B_{i}} \equiv B_{i}\left(y+\delta y, v_{1}+\delta v_{1}\right), \Delta B_{i} \equiv B_{i}\left(y, v_{1}+\delta v_{1}\right)-\lambda_{i}\left(y, v_{1}\right), i=\overline{1, n}, i=\overline{1, n}$, $\Delta f \equiv f\left(x, t, v_{2}+\delta v_{2}\right)-f\left(x, t, v_{2}\right)$.

Let $\eta_{h}(x, t)=\frac{1}{h} \int_{t-h}^{t} \bar{\eta}(x, \tau) d \tau, 0<h<\tau$ where $\bar{\eta}=\delta y(x, t)$ at $(x, t) \in \Omega_{t_{1}}$, zero at $t>t_{1}\left(t_{1} \leq T-h\right)$ and $\Omega_{t_{1}}=D \times\left(0, t_{1}\right]$. In identity (5) put $\eta(x, t)$ instead of $\eta_{h}(x, t)$, and following the method in [11,p. 166-168] we obtain

$$
\begin{gathered}
\frac{1}{2} \int_{\mathrm{D}}(\delta y)^{2} \mathrm{~d} x+\int_{\Omega_{\mathrm{t}_{1}}}\left[\sum_{i=1}^{n} \overline{\lambda_{i}}\left(\frac{\partial \delta y}{\partial x_{i}}\right)^{2}+\sum_{i=1}^{n} \frac{\partial \lambda_{i}\left(y+\theta_{1 i}, v_{0}+\delta v_{0}\right)}{\partial y} \frac{\partial y}{\partial x_{i}} \frac{\partial \delta y}{\partial x_{i}} \delta y\right] d x d t \\
+\int_{\Omega_{\mathrm{t}_{1}}} \sum_{i=1}^{n} \Delta \lambda_{i} \frac{\partial y}{\partial x_{i}} \frac{\partial \delta}{\partial x_{i}} \mathrm{~d} x d t+\sum_{i=1}^{n} \frac{\partial B_{i}\left(y+\theta_{2 i}, v_{1}+\delta v_{1}\right)}{\partial y} \frac{\partial y}{\partial x_{i}}(\delta y)^{2} d x d t \\
+\int_{\Omega_{\mathrm{t}_{1}}} \sum_{i=1}^{n} \overline{B_{i}} \frac{\partial \delta y}{\partial x_{i}} \delta y+\int_{\Omega_{\mathrm{t}_{1}}} \sum_{i=1}^{n} \Delta B_{i}\left(\frac{\partial y}{\partial x_{i}}\right) \delta y d x d t-\int_{\Omega_{\mathrm{t}_{1}}} \Delta f \delta y d x d t=0
\end{gathered}
$$

Hence,from the above assumptions and applying Cauchy Bunyakoviskii inequality, we obtain

$$
\begin{gathered}
\frac{1}{2} \int_{\mathrm{D}}\left(\delta \mathrm{y}\left(x, \mathrm{t}_{1}\right)^{2} \mathrm{~d} x+v_{0} \int_{\Omega_{\mathrm{t}_{1}}} \sum_{i=1}^{n}\left|\frac{\partial \delta y}{\partial x_{\mathrm{i}}}\right|^{2} \mathrm{~d} x \mathrm{dt}\right. \\
\leq\left(\mathrm{C}_{3}+\mathrm{C}_{4}\right)\left(\int_{\Omega_{\mathrm{t}_{1}}} \sum_{i=1}^{n}\left|\frac{\partial \delta y}{\partial x_{i}}\right|^{2} \mathrm{~d} x \mathrm{dt}\right)^{\frac{1}{2}}\left(\int_{\Omega_{\mathrm{t}_{1}}}(\delta \mathrm{y}(x, \mathrm{t}))^{2} \mathrm{~d} x \mathrm{dt}\right)^{\frac{1}{2}} \\
+\left\{\int_{\Omega_{\mathrm{t}_{1}}} \sum_{i=1}^{n}\left|\Delta \lambda_{i} \frac{\partial y}{\partial x_{i}}\right|^{2} \mathrm{~d} x \mathrm{dt}\right\}^{\frac{1}{2}}\left(\int_{\Omega_{\mathrm{t}_{1}}} \sum_{i=1}^{n}\left|\frac{\partial \delta y}{\partial x_{i}}\right|^{2} \mathrm{~d} x \mathrm{dt}\right)^{\frac{1}{2}}+\mathrm{C}_{5} \int_{\Omega_{\mathrm{t}_{1}}}(\delta \mathrm{y}(x, \mathrm{t}))^{2} \mathrm{~d} x \mathrm{dt} \\
-\int_{0}^{\mathrm{t}_{1}}\left\{\int_{\mathrm{D}}\left|\Delta \mathrm{f}-\sum_{i=1}^{n} \Delta \mathrm{B}_{i}\left(\frac{\partial y}{\partial x_{\mathrm{i}}}\right)\right|^{\frac{1}{2}} \mathrm{~d} x\left(\int_{\mathrm{D}}(\delta \mathrm{y})^{2} \mathrm{~d} x\right)^{\frac{1}{2}}\right\} \mathrm{dt},
\end{gathered}
$$

where $C_{3}, C_{4}, C_{5}$ are positive constants not depending on $\delta v$.

Applying Cauchy's inequality with $\varepsilon$ and combine similar terms, then multiply both sides by two, we obtain

$$
\begin{aligned}
& \left\|\delta y\left(x, t_{1}\right)\right\|_{L_{2}(D)}^{2}+\frac{v_{0}}{2}\left\|\sum_{i=1}^{n} \frac{\partial \delta y}{\partial x_{i}}\right\|_{L_{2}\left(\Omega_{t_{1}}\right)}^{2} \leq C_{6}\|\delta y(x, t)\|_{L_{2}\left(\Omega_{t_{1}}\right)}^{2} \\
& +2\left\{\int_{\Omega_{t_{1}}} \sum_{i=1}^{n}\left|\Delta \lambda_{i} \frac{\partial y}{\partial x_{i}}\right|^{2} d x d t\right\}^{\frac{1}{2}}\left\|\sum_{i=1}^{n} \frac{\partial \delta y}{\partial x_{i}}\right\|_{L_{2}\left(\Omega_{t_{1}}\right)}^{2} \\
& +2 \max _{0 \leq \tau \leq t_{1}} \| \delta y\left(x, \tau \|_{L_{2}(D)} \int_{0}^{t_{1}}\left\{\int_{D}\left|\Delta f-\sum_{i=1}^{n} \Delta B_{i}\left(\frac{\partial y}{\partial x_{i}}\right)\right|^{2} d x\right\}^{\frac{1}{2}} d t\right.
\end{aligned}
$$

Now we replace

$$
y\left(t_{1}\right)=\max _{0 \leq \tau \leq t_{1}} \| \delta y\left(x, \tau\left\|_{L_{2}(D)},\right\| \delta y(x, t) \|_{L_{2}\left(\Omega_{t_{1}}\right)}^{2}=t_{1}\left(y\left(t_{1}\right)\right)^{2} .\right.
$$


This gives us the inequality

$$
\begin{aligned}
& \left\|\delta y\left(x, t_{1}\right)\right\|_{L_{2}(D)}^{2}+\frac{v_{0}}{2}\left\|\sum_{i=1}^{n} \frac{\partial \delta y}{\partial x_{i}}\right\|_{L_{2}\left(\Omega_{t_{1}}\right)}^{2} \leq C_{6} t_{1}\left(y\left(t_{1}\right)\right)^{2} \\
& \quad+2\left\{\int_{\Omega_{t_{1}}} \sum_{i=1}^{n}\left|\Delta \lambda_{i} \frac{\partial y}{\partial x_{i}}\right|^{2} d x d t\right\}^{\frac{1}{2}}\left\|\sum_{i=1}^{n} \frac{\partial \delta y}{\partial x_{i}}\right\|_{L_{2}\left(\Omega_{t_{1}}\right)}^{2} \\
& \quad+2 y\left(t_{1}\right) \int_{0}^{t_{1}}\left\{\int_{D}\left|\Delta f-\sum_{i=1}^{n} \Delta B_{i}\left(\frac{\partial y}{\partial x_{i}}\right)\right|^{2} d x\right\}^{\frac{1}{2}} d t \equiv j\left(t_{1}\right) .
\end{aligned}
$$

From this follows the two inequalities

$$
\left(y\left(t_{1}\right)\right)^{2} \leq j\left(t_{1}\right)
$$

and

$$
\left\|\sum_{i=1}^{n} \frac{\partial \delta y}{\partial x_{i}}\right\|_{L_{2}\left(\Omega_{t_{1}}\right)} \leq \frac{2}{v_{0}} j\left(t_{1}\right)
$$

We take the square root of both sides of (12) and (13), add together the resulting inequalities and then majorize the right-hand side in the same way in [12] (pp. 117-118) and this proves the estimate (6). This completes the proof of the theorm.

Corollary 3.1 Under the above assumptions, the right part of estimate (6) converges to zero at $\sum_{m=0}^{2}\left\|\delta v_{m}\right\|_{l_{2}} \rightarrow 0$, therefore

$$
\|\delta y\|_{v_{2}^{1}, o(\Omega)} \rightarrow 0 \text { at } \sum_{m=0}^{2}\left\|\delta v_{m}\right\|_{l_{2}} \rightarrow 0
$$

Hence from the theorem on trace [13] we get

$$
\|\delta y\|_{L_{2}(\Omega)} \rightarrow 0,\|\delta y\|_{L_{2}(S)} \rightarrow 0 \text { at } \sum_{m=0}^{2}\left\|\delta v_{m}\right\|_{l_{2}} \rightarrow 0
$$

Now we consider the functional $J_{0}(v)=\int_{S}\left[y(\zeta, t)-f_{0}(\zeta, t)\right]^{2} d \zeta d t$.

Theorem 3.2 The functional $\mathrm{J}_{0}(v)$ is continuous on $\mathrm{V}$.

proof

Let $\delta v=\left(\delta v_{0}, \delta v_{1}, \delta v_{2}\right), \delta v_{\mathrm{m}} \in \mathrm{l}_{2}, \mathrm{~m}=\overline{0,2}$ be an increment of control on an element $v \in \mathrm{V}$ such that $v+\delta v \in \mathrm{V}$. For the increment of $\mathrm{J}_{0}(v)$ we have

$$
\Delta \mathrm{J}_{0}(v)=\mathrm{J}_{0}(v+\delta v)-\mathrm{J}_{0}(v)=2 \int_{S}\left[\mathrm{y}(\zeta, \mathrm{t})-\mathrm{f}_{0}(\zeta, \mathrm{t})\right] \delta \mathrm{y}(\zeta, \mathrm{t}) \mathrm{d} \zeta \mathrm{d} \mathrm{t}+\int_{S}[\delta \mathrm{y}(\zeta, \mathrm{t})]^{2} \mathrm{~d} \zeta \mathrm{dt}
$$


Applying the Cauchy-Bunyakovskii inequality, we obtain

$$
\left|\Delta \mathrm{J}_{0}(v)\right| \leq 2\left\|\mathrm{y}(\zeta, \mathrm{t})-\mathrm{f}_{\mathrm{O}}(\zeta, \mathrm{t})\right\|_{\mathrm{L}_{2}(S)}\|\delta \mathrm{y}(\zeta, \mathrm{t})\|_{\mathrm{L}_{2}(S)}+\|\delta \mathrm{y}(\zeta, \mathrm{t})\|_{\mathrm{L}_{2}(S)}^{2}
$$

An application of the Corollary 3.1 completes the proof.

Theorem 3.3 For any $\beta \geq 0$ the problem (1)-(4) has a least one solution.

proof

The set of $V$ is closed and bounded in $l_{2}$. Since $J_{0}(v)$ is continuous on $V$ by Theorem 3.2, so is

$$
\mathrm{J}_{\beta}(v)=\mathrm{J}_{0}(v)+\beta \sum_{\mathrm{m}=0}^{2}\left\|v_{\mathrm{m}}-w_{\mathrm{m}}\right\|_{\mathrm{l}_{2}}^{2} .
$$

Then from the Weierstrass theorem [14] it follows that the problem (1)-(4) has a least one solution. This completes the proof of the theorm.

\section{The Uniqueness Theorem}

According to the above discussions, we ca easily obtain a theorem concerning solution uniqueness for the considering optimal control problem (1)-(4).

Theorem 4.1 There exists a dense set $K$ of $l_{2}$ such that for any $\omega_{m} \in K, m=\overline{0,2}$ the problem (1)-(4) for $\beta>0$ has a unique solution.

proof The functional $\mathrm{J}_{0}(v)$ is bounded below, and the foreging establishes that it is continues on $\mathrm{V}$. Furthermore, $\mathrm{l}_{2}$ is uniformaly convex [12]. It thus follows from a theorm in [16] that the space $l_{2}$ contains an everywhere-dense subset $K$ such that the problem (1)-(4) has a unque solution when $\omega_{m} \in K, m=\overline{0,2}$ and $\beta>0$. This completes the proof of the theorm.

\section{Conclusion}

We have investigated a constrained optimal control problems governed by quasilinear parabolic equations with controls in the coefficients of the equation. The existence and uniqueness of the optimal control problem is proved. 


\section{Acknowledgment}

The authers gratefully acknowledgment the referee, who made useful suggestions and remarks which helped to improve the paper.

Received: September 2011. Accepted: September 2012.

\section{References}

[1] Li Chun-fa, Xue Yang and En-min Feng,Optimal control problem governed by semilinear parabolic equation and its algorithm,Acta Mathematicae Sinica,186,2008,29-40.

[2] D. A. Lashin,on the existence of optimal control of temperature regimes, J. of Math. Sciences, $158(2), 2009$.

[3] I. Chryssoverghi,Mixed discretization-optimization methods for relaxed optimal control of nonlinear parabolic systems,Proc. of 6th WSEAS (Int. Conf.), Lishbon, 2006,22-41.

[4] M. H. Farag, On the derivation of discrete conjugate boundary value problem for an optimal control parabolic problem, New Zeal J. of Maths, 32,3003, 21-31.

[5] T. B. Gardashov, Solution of inverse problems for the quasilinear heat conduction equation for the multidimensional case, J. Engrg. Phys., 61(3),1991,472-478.

[6] A. D. Iskenderov and R. K. Tagiev, Optimization problems with controls in coefficients of parabolic equations,Differentsialnye Uravneniya,19(8),1983,1324-1334.

[7] M. H. Farag,A stability theorem for constrained optimal control problems, Journal of Computational Mathematics, V. 22(5),2006,635-640.

[8] M. H. Farag,Computing optimal control with a quasilinear parabolic partial differential equation, Surveys in Mathematics and its Applications,23,2009,34-44.

[9] A. N. Tikhonov and N. Ya. Arsenin, Methods for the solution of incorrectly posed problems, Nauka, Moscow, Russian,1974.

[10] F. Murat,Contre-examples pour dives problems ou le controle intervient dans les coefficients,Ann. Mat. Pure Appl.,112,1977,49-68.

[11] O. A. Ladyzhenskaya, V. A. Solonnikov and N. N. Ural'tseva,Linear and quasilinear parabolic equations,Nauka, Moscow, Russian,1976.

[12] O. A. Ladyzhenskaya,Boundary value problems of mathematical physics ,Nauka, Moscow, Russian,1973. 
[13] V. P. Mikhailov,Partial differential equations,Nauka, Moscow, Russian,1983.

[14] W. Krabs,Optimization and Approximation,Wiley, New York,1979.

[15] K. Yosida,Functional analysis, Mir, Moscow, 1967.

[16] M. Goebel,On existence of optimal control,Math. Nuchr.,93,1979,67-73. 